CRÍTICA, Revista Hispanoamericana de Filosofía

Vol. XXV, No. 75 (diciembre 1993): 3-29

\title{
HISTORIA Y FILOSOFÍA EN LA INTERPRETACIÓN DE LAS TEORÍAS POLÍTICAS
}

\author{
Ambrosio Velasco Gómez \\ Instituto de Investigaciones Filosóficas
}

UNAM

\section{Introducción}

Desde la crítica popperiana al positivismo lógico, la filosofía de la ciencia tiende a vincularse cada vez más con la historia de la ciencia. La tesis de Karl R. Popper de que "todo intento de comprender una teoría se ve abocado a abrir una investigación histórica en torno a dicha teoría"l cobra un amplio consenso entre filósofos e historiadores de la ciencia en nuestros días.

Sin embargo, este consenso es el punto de partida de un nuevo y amplio debate. Si bien hay acuerdo en que el estudio de la historia de las teorías científicas es indispensable para su adecuada comprensión filosófica, persiste una intensa discusión sobre cómo interpretar la historia de la ciencia (y en general la historia) y cómo se vincula ésta con la filosofía de la ciencia. Muestra de este intenso debate es la polémica entre Karl R. Popper y Thomas Kuhn² que

${ }^{1}$ K.R. Popper, Conocimiento objetivo, Tecnos, Madrid, 1979, p. 168.

${ }^{2}$ Véase I. Lakatos y A. Musgrave, Crítica y desarrollo del conocimiento, Grijalbo, 1968. 
desde los años sesenta ha continuado y generado un diálogo cada vez más complejo. ${ }^{3}$

En esta discusión no sólo se tienen concepciones distintas sobre las teorías científicas y su desarrollo, sino también diferentes perspectivas que defienden distintos modos de interpretación histórica.

Para filósofos como K.R. Popper la historia de las ciencias debe ajustarse a un principio universal de racionalidad: resolución de problemas por medio de conjeturas y superación continua de éstas mediante refutaciones. Para historiadores como Kuhn, la aceptación de este principio conduce a deformaciones históricas inaceptables. Desde este punto de vista, no hay que presuponer un criterio de cientificidad, sino más bien derivar la noción de cientificidad del estudio objetivo sobre cómo los científicos se han comportado a lo largo de la historia.

Aunque Imre Lakatos y posteriormente Larry Laudan y Mary Hesse han propuesto diferentes modelos para sintetizar e integrar filosofía e historia, la formulación de una perspectiva histórico-filosófica para el estudio de la ciencia que obtenga consenso está aún lejos de realizarse. Este debate se vuelve todavía más complejo con la participación de sociólogos y filósofos con orientaciones sociológicas que demandan una mayor atención a los aspectos sociales del desarrollo científico. ${ }^{4}$

3 Una propuesta original que integra diferentes dimensiones de la complejidad de esta discusión es la de Mary Hesse en su libro Revolutions and Reconstructions in the Philosophy of Science, The Harvester Press, 1980. Un análisis sucinto y claro de las principales concepciones que se han generado en esta discusión lo presenta Ana Rosa Pérez Ransanz en su ensayo "Modelos del cambio científico", en Enciclopedia iberoamericana de filosofía, vol. IV, La ciencia: estructura y desarrollo, Ulises Moulines (comp.), Editorial Trota, CSIC, V Centenario, Madrid, 1993, pp. 181-202.

4 Para una presentación y discusión detallada de los diferentes programas en sociología de la ciencia, véase León Olivé (comp.), La 
Este complejo y persistente debate no es exclusivo de la filosofía de la ciencia, sino que también se manifiesta en otras áreas de la filosofía. ${ }^{5}$ En particular en el ámbito de la filosofía política este debate ha tomado especial relevancia; se ha desarrollado en sus propios términos, pero ha mostrado analogías y similitudes con el debate en el campo de la filosofía de la ciencia. Así por ejemplo, tanto en el estudio de la historia de las teorías políticas, como en el campo de la filosofía de la ciencia, existen posturas filosóficas que defienden la existencia de criterios universales para evaluar las teorías (Strauss, Popper) a las cuales se oponen perspectivas historiográficas (Skinner, Kuhn) que conciben las teorías ligadas siempre a criterios contextuales. Y del mismo modo en que autores como Lakatos y Laudan tratan de realizar una mediación integradora en la polémica de la filosofía de la ciencia, también en la discusión sobre las intepretaciones y reconstrucciones de las teorías políticas, autores como Alasdair MacIntyre proponen integrar aspectos de las perspectivas de historiadores y de filósofos, a fin de poder comprender adecuadamente la naturaleza de las teorías políticas. Estas analogías en los debates entre historiadores y filósofos de la ciencia por un lado, e historiadores y filósofos políticos, por otro, muestran que hay tanto un consenso respecto a la necesidad de integrar la historia y la reflexión filosófica, como una persistencia de problemas centrales acerca de cómo realizar tal integración. Dada la existencia de estos paralelismos, es de esperarse que los avances realizados en uno de estos campos tengan

explicación social del conocimiento, FCE, México, 1985 (especialmente la introducción); id., Conocimiento, sociedad y realidad, Siglo XXI, México, 1988.

${ }^{5}$ Una interesante colección de artículos sobre la relación entre historia y filosofía en diferentes ámbitos se encuentra en R. Rorty, J.B. Schneewind y Q. Skinner (comps.), Philosophy in History, Cambridge University Press, 1984. 
un importante valor heurístico en el otro. En este trabajo se estudiará solamente el debate entre filósofos e historiadores de la teoría política.

En el ámbito del estudio de la teoría política, las formas en que los filósofos políticos y los historiadores abordan el problema de la relación historia-filosofía difieren de manera radical. Desde una perspectiva tradicionalmente filosófica, autores como Leo Strauss, Sheldon Wolin y Hannah Arendt, entre otros, conciben la historia de las teorías políticas como una fuente de problemas para la reflexión filosófica. Para ellos la historia es una disciplina instrumental y está subordinada a la reflexión filosófica. Por otro lado, desde una visión más afín a la tradición historiográfica, autores como Quentin Skinner, John Dunn y J.G.A. Pocock defienden la autonomía de la historia con relación a la filosofía política, y argumentan que la forma en que los filósofos políticos straussianos han interpretado la historia de las ideas políticas lleva a una distorsión de lo que efectivamente han sido las teorías políticas. ${ }^{6}$ Estos representantes de "la nueva historia de las ideas políticas" han desarrollado una perspectiva de interpretación basada en la filosofía del lenguaje de Wittgenstein y Austin. Inversamente a lo que sucede con Strauss y sus seguidores, los representantes de "la nueva historia de las ideas" vinculan la filosofía del lenguaje de una manera instrumental para elaborar una metodología de análisis histórico.

Debido a la falta de una perspectiva que integre la reflexión filosófica y el análisis histórico, los enfoques de historiadores como Skinner y de filósofos como Strauss conducen a dilemas que obstaculizan una adecuada comprensión de las teorías políticas.

6 Para una comparación de las posiciones de los filósofos tradicionales y los nuevos historiadores de la teoría política, véase J.G. Gunnell, Political Theory. Tradition and Interpretation, Little Brown, Boston, 1979. 
Este trabajo se propone examinar brevemente los planteamientos de Leo Strauss y Quentin Skinner, ${ }^{7}$ discutir los dilemas a los que estas perspectivas conducen y proponer algunas vías para integrar aspectos de éstas, de tal manera que puedan superarse los dilemas planteados. Para explicar esta propuesta reconstructiva, me apoyaré en algunas tesis de Alasdair MacIntyre.

\section{La perspectiva filosófica de Strauss}

Strauss considera que el estudio de la historia de las teorías políticas es indispensable para la filosofía política. Para Strauss, la historia tiene la función de rescatar con la mayor fidelidad las teorías y tradiciones políticas circunscritas en contextos históricos específicos. Una vez recobrada esta información histórica, es trabajo del filósofo escudriñarla críticamente en busca de respuestas verdaderas a los problemas fundamentales de la filosofía política, tales como, ¿cuál es la naturaleza de lo político?, ¿cuál es el orden político justo?

Parecería que, a primera vista, Strauss delimita bien los campos de la filosofía política y de la historia de las teorías políticas. Pero bien mirada, la cosa es diferente. Para Strauss la correcta interpretación de la historia de la teoría política implica entender el texto de acuerdo con lo que el autor originalmente se propuso comunicar. En este sentido,

7 Claro está que con la discusión de cada uno de ellos no se agota, de ninguna manera, el análisis de las perspectivas que, junto con otros autores, han contribuido a formar. Más allá de tesis fundamentales que los filósofos políticos, por un lado (Strauss, Wolin, Arendt, entre otros) e historiadores de las ideas políticas (Skinner, Pocock, Dunn), por otro, existen importantes diferencias dentro de cada uno de estos grupos. Por ejemplo: un diferencia importante entre Strauss y Arendt se refiere a las distintas posiciones de estos autores respecto a la importancia de la filosofía política antigua. Mientras que Strauss la tiene en gran estima, Arendt aprecia más la actividad política misma en las repúblicas antiguas. 
Strauss parece coincidir con los historiadores en cuanto a que se oponen a interpretaciones anacrónicas basadas en una ingenua creencia del progreso continuo en la historia de las ideas (presentismo y progresismo). ${ }^{8}$ Para ello, el proceso de lectura del texto debe tener presente la estrategia que el autor siguió al escribirlo.

Strauss considera que todo gran autor de teoría política tiene o tuvo como propósito ofrecer una respuesta verdadera a los problemas fundamentales de la filosofía política. Este esfuerzo del filósofo político necesariamente lo lleva a trascender el ámbito de sus circunstancias inmediatas y particularidades históricas. El resultado de esta trascendente reflexión filosófica es justamente un conocimiento teórico en sentido estricto, que difiere y se contrapone a las opiniones y creencias políticas particulares del tiempo del autor (ideología). ${ }^{9}$

Strauss sostiene que la oposición y crítica a las opiniones dominantes del momento, a la que necesariamente arriba todo gran autor de teoría política, representa un elemento de disolución social y política. Por lo tanto, siguiendo una tesis eminentemente socrática, Strauss considera que el filósofo político constituye un peligro para la integridad y conservación del orden social y político de su tiempo. A fin de poder dedicarse a la búsqueda de la verdad, sin

8 "La tarea del historiador de las ideas es comprender a los pensadores del pasado exactamente como ellos se comprendieron a sí mismos o, en otras palabras, revitalizar su pensamiento de acuerdo con su propia y original interpretación." (L. Strauss, "Political Philosophy and History", en What is Political Philosophy? and Other Studies, Free Press, Glencone (Ill.), 1959, p. 67.)

9 “El filósofo está en última instancia comprometido a trascender no solamente la dimensión de la opinión común sino también la dimensión de la vida política misma." (L. Strauss, "On Classical Political Philosophy", en Rebirth of Classical Political Philosophy, editado por T. Pangle, University of Chicago Press, Chicago, 1989, p. 60.) 
poner en riesgo la estabilidad política de su estado (y con ello su misma integridad física), todo gran autor de filosofía política tiene que desarrollar una estrategia comunicativa singular. El texto de teoría política debe contener un doble mensaje: por un lado, un mensaje "exotérico", que es público, fácilmente comprensible, superficial e inocuo, y que tiende a repetir y justificar las opiniones políticas dominantes. Por otro lado, el autor debe ser capaz de comunicar en forma encubierta, entre líneas, el mensaje profundo, crítico y verdaderamente filosófico que intenta responder a problemas fundamentales de la política y que pretende tener validez universal. Este segundo mensaje que Strauss denomina "esotérico" está inscrito en un código que sólo el atento lector filosófico puede descifrar y entender. ${ }^{10}$

Para Strauss, la historia de la teoría política debe rescatar el mensaje esotérico que contiene las enseñanzas profundas y serias del autor. Para ello, el historiador debe adoptar una actitud filosófica y trascender el nivel superficial de las opiniones históricamente circunscritas de los textos (nivel exotérico) y acceder al nivel profundamente filosófico, al nivel de las respuestas teóricas universales a problemas fundamentales, que constituye el significado esotérico de los textos y que sólo puede ser rescatado mediante una lectura entre líneas. ${ }^{11}$

10 Cfr. L. Strauss, "On a Forgotten Kind of Writing", en What is Political Philosophy? and Other Studies (especialmente pp. 21-22).

11 En este sentido, Strauss estaría de acuerdo con Popper al considerar que los contenidos teóricos de la filosofía y de la ciencia constituyen un "tercer mundo", el cual escapa a las efímeras creencias y experiencias personales (segundo mundo) y a las contingencias de los contextos históricos. Strauss también estaría de acuerdo con Popper en que la interpretación de la historia de las teorías políticas (o filosóficas o científicas) es un trabajo internalista. Tratar de realizar reconstrucciones externalistas de los contenidos estrictamente teóricos implicaría una confusión entre el mensaje "exotérico" (susceptible de interpretación y explicación sociológica y contextual) y el mensaje "esotérico" 
Desde esta visión straussiana, la aparente independencia entre historia y filosofía de la teoría política desaparece. Para desarrollar su función de rescatar el significado original de los textos de teoría política, el historiador tiene que conocer de antemano cuáles son los problemas filosóficos fundamentales a los que todo autor de teoría política intenta dar respuesta. De esta manera, la historia de la teoría política no sólo resulta instrumental para la filosofía política, sino que se vuelve completamente dependiente de ella. Lejos de ser una integración crítica entre historia y filosofía política, la propuesta straussiana es una subsunción de la historia en la filosofía. En suma, el historiador de la teoría política debe "llevar a cabo una transformación o conversión en filósofo si quiere realizar su trabajo correctamente, si quiere ser un historiador de la filosofía". ${ }^{12}$

\section{La perspectiva historicista de Skinner}

Quentin Skinner y los practicantes de "la nueva historia de las ideas" 13 critican la pretensión straussiana de considerar la historia de las teorías políticas como instrumental y dependiente de la filosofía política. Los seguidores de esta perspectiva historiográfica empiezan por negar la persistencia de problemas fundamentales a lo largo de la historia de las teorías políticas y afirman, por el contrario, que toda

(que por su naturaleza trascendente no puede explicarse en términos sociológicos).

12 L. Strauss, "How to Begin the Study of Medieval Philosophy?", en The Rebirth of Classical Political Philosophy, p. 211.

13 La escuela de Dunn, Skinner y Pocock es denominada de diferentes maneras. David Boucher se refiere a ellos como "nuevos historiadores del pensamiento político" o "revisionistas". (Cfr. D. Boucher, "New Histories of Political Thought for Old", Political Studies, vol. XXXI, 1983, pp. 112-121.) Gunnell se refiere a esta escuela como "la nueva historia de las teorías políticas". (Cfr. Gunnell, op. cit.) 
teoría política responde a problemas prácticos y específicos de su contexto histórico.

En particular, Skinner desarrolla esta visión historicista basado en Wittgenstein y Austin, por un lado, y en Collingwood, por otro. De los dos primeros Skinner construye un modelo del significado de los textos, y del segundo elabora un modelo del papel que desempeñan los textos de teoría política y en general el discurso escrito en el cambio social. Veamos cada uno de estos modelos.

Skinner considera que todo texto de teoría política fue escrito por su autor con el propósito de influir en el clima ideológico-político de su tiempo. Desde este punto de vista, a diferencia de Strauss, los textos de teoría política son esencialmente ideologías políticas en acción. ${ }^{14}$ En cuanto ideologías, las teorías políticas no son falsas o verdaderas, sino efectivas o no para justificar, cuestionar o transformar las creencias, actitudes y valores dominantes. En consecuencia, la historia de las ideas políticas no es dependiente de presupuestos filosóficos que señalan cuáles son los problemas fundamentales a los que las teorías responden y los criterios morales y epistemológicos universales con los que hay que evaluar las respuestas. Además, la historia no puede tener una función instrumental para la filosofía política como la entiende Strauss; la historia no es una disciplina que se presenta ante los tribunales filosóficos para ser juzgada moral o epistemológicamente, no tiene la función, en términos de Skinner, de un "juez ejecutor" (hanging judge), sino que, más bien, tiene simplemente la función de un ángel que rescata y transcribe (recording angel) el

14 Cfr. Q. Skinner, "Some Problems in the Analysis of Political Thought and Action", en Tully James y Q. Skinner (comps.), Meaning and Context: Quentin Skinner and his Critics, Princeton University Press, Princeton (N.J.), 1988. Véase también en esta excelente antología de textos de y sobre Skinner su artículo "Reply to My Critics". 
significado original de los textos políticos en su contexto histórico específico. ${ }^{15}$

El modelo del significado que Skinner deriva de Wittgenstein, Austin y secundariamente de Frege y el emotivismo ético (Stevenson), constituye el recurso metodológico fundamental para que el historiador pueda no sólo rescatar el significado original del texto, sino también dar cuenta de las funciones ideológicas que desempeñó en su tiempo. ${ }^{16}$

Para Skinner, el significado de cualquier acto lingüístico, y en particular el de la escritura de un texto, está determinantemente vinculado a las intenciones primarias que el autor tuvo al escribir el libro. Skinner identifica dicha intencionalidad con la fuerza ilocucionaria del acto o actos lingüísticos que constituyen el texto. Dicha fuerza ilocucionaria, si bien está internamente correlacionada con la ejecución misma (escritura del texto) y con su significado, apunta siempre hacia afuera del texto, hacia el entorno ideológico. Por esta razón, para Skinner una mera interpretación textualista o meramente contextualista es insuficiente. Ambos tipos de análisis son indispensables para una correcta interpretación del texto.

No obstante la importancia que Skinner asigna a la fuerza ilocucionaria, él mismo advierte que la identificación de ésta no es suficiente para comprender un texto político en sus términos originales. Para ello también es indispensable reconstruir el significado del léxico utilizado por el autor, de acuerdo con el uso y las convenciones lingüísticas de la época. Siguiendo a Frege, Skinner distingue dos com-

${ }^{15}$ Cfr. Q. Skinner, Machiavelli, Oxford University Press, Oxford, 1985, p. 88.

${ }^{16}$ Este modelo lo desarrolla Skinner principalmente en sus artículos "Some Problems in the Analysis of Political Thought and Action", "Hermeneutics and the Role of History", "Motives, Intentions and Interpretation" y "Reply to My Critics". Todos estos trabajos forman parte de su libro Meaning and Context. 
ponentes fundamentales del significado: sentido y referencia. Sobre esta concepción del significado, Skinner añade la distinción emotivista del sentido descriptivo y sentido evaluativo; este último tipo de sentido es determinante sobre la fuerza ilocucionaria.

El autor puede darle a su texto una determinada función ideológica mediante la manipulación del sentido descriptivo o del sentido evaluativo de los conceptos claves de una teoría política. Manteniendo o cambiando el sentido descriptivo de conceptos claves como libertad, democracia, virtud, crueldad, etc., el autor puede inducir en su comunidad una justificación o, por el contrario, un cuestionamiento de creencias y percepciones políticas. Piénsese por ejemplo, en el cambio del sentido descriptivo del concepto republicano de democracia como lo entiende Rousseau o Tocqueville, por el concepto liberal de democracia como lo proponen autores norteamericanos como Berelson y Dahl. Este cambio induce a concebir regímenes políticos que serían tiránicos en la teoría republicana, como democracias, con la connotación valorativa positiva que el término conserva.

Por otra parte, al operar sobre el sentido evaluativo de términos claves, y con ello sobre su fuerza ilocucionaria, el autor afecta las actitudes y valores de su comunidad. Piénsese, por ejemplo, en la forma como Hobbes maneja las connotaciones valorativas del concepto de libertad, a fin de justificar el absolutismo en aras de la seguridad; o bien en el cambio de las connotaciones valorativas de los conceptos de crueldad, temor, amor, clemencia, fuerza y astucia en el pensamiento político de Maquiavelo.

Para Skinner, este modelo interpretativo de textos es la herramienta fundamental para que el historiador de las ideas políticas identifique el papel ideológico de un texto en su contexto original y recupere su auténtico significado. 


\section{Anacrónicos o anticuarios: algunos dilemas}

Como se ha visto, Strauss considera la historia como dependiente de presupuestos de filosofía política y como mera proveedora de información sobre el pasado, mientras que Skinner restringe el papel de la filosofía del lenguaje y de la historia a mera herramienta de apoyo metodológico para la interpretación de textos del pasado. Estas concepciones de la relación entre historia y filosofía política no conducen a una adecuada integración de la reflexión filosófica y la reconstrucción histórica en la explicación y comprensión de la naturaleza, función y desarrollo de las teorías políticas. Estas dos perspectivas, lejos de llegar a un fructífero encuentro, simplemente se refutan recíprocamente y con ello ahondan la separación entre filosofía e historia de las teorías políticas. Esta divergencia conduce al estudioso de las teorías políticas a los siguientes dilemas.

Si miramos bien la función que cada una de estas perspectivas asigna a la reflexión filosófica crítica, encontramos que, en el caso de Strauss, esta función se aplica a los contenidos sustantivos de las teorías políticas. Para Strauss la relevancia del estudio de la historia de las teorías políticas, entendida como la historia de respuestas a problemas fundamentales, radica en la posibilidad de compararla y evaluarla de acuerdo con criterios filosóficos universales. Si la historia no alcanza este nivel crítico y evaluativo, entonces la historia como disciplina es mera actividad de anticuarios, más que una actividad filosófica.

Para Skinner, esta manera filosófica de interpretar los textos de teoría política en términos de su contribución a la solución de problemas supuestamente universales, implica distorsionar la historicidad específica de las teorías políticas y hacer interpretaciones anacrónicas de teorías del pasado. Este tipo de anacronismos trae consigo dos desventajas 
más. Primero, al perder de vista la historicidad específica de las teorías del pasado, se pierde con ello la función política e ideológica que dicha teoría desempeñó. El segundo problema del anacronismo es que las interpretaciones del pasado, siguiendo la estrategia de leer entre líneas, resultan como bien lo señala J.G.A. Pocock, irrefutables (y desde luego inverificables) en términos de evidencia histórica, ${ }^{17}$ ya que siempre puede aludirse a una lectura entre líneas, de la cual no hay evidencia para forzar la interpretación de un texto.

Estos dos problemas son superados de manera adecuada por la perspectiva skinneriana, gracias precisamente a la atención que se pone en el método de interpretación. El modelo de la relación entre texto, acto lingüístico y significado intencional permite a Skinner dar cuenta de la historicidad de las teorías políticas y de la función ideológica específica que desempeñan. Además, la metodología skinneriana propone una forma para juzgar la validez de las interpretaciones en términos de evidencia histórica.

Sin embargo, la perspectiva skinneriana adolece de ciertos problemas: al insistir en una metodología encaminada exclusivamente a dar cuenta de la historicidad específica de la obra y del papel ideológico que desempeñó en sus circunstancias originales, se pierden de vista las funciones cognoscitivas de la teoría en cuestión, así como el problema relativo a la validez de las teorías mismas en cuanto conocimiento de la realidad política.

Así, desde la perspectiva skinneriana, el estudioso de la historia de las teorías políticas está imposibilitado para juzgar la validez de teorías del pasado y sobre todo su relevancia para el presente. En su ardua crítica de interpre-

17 Cfr. J.G.A. Pocock, "Prophet and Inquisitor", Political Theory, noviembre, 1975. 
taciones anacrónicas, Skinner parece ubicarse en el trabajo del anticuario, preocupado por el rescate de la originalidad histórica de la obra, pero carente de criterios para juzgar su relevancia para el presente.

Si comparamos las ventajas y desventajas de la perspectiva (anacrónica) de Strauss y la perspectiva (anticuaria) de Skinner, no podemos optar por alguna de ellas en particular sin sufrir los dilemas a los que conducen. Si optamos por la perspectiva straussiana podríamos discutir la validez de las teorías políticas de acuerdo con criterios racionales, pero no la validez de sus interpretaciones y reconstrucciones; se podrían analizar sus contenidos cognoscitivos, pero no sus funciones ideológicas; podríamos imputar relevancia actual a los textos, pero no aproximarnos a su significado auténtico y original.

Por el contrario, si optamos por la perspectiva de Skinner, sería posible evaluar la validez de interpretaciones alternativas sobre un texto de teoría política, pero no podríamos juzgar la validez de las mismas teorías políticas, y mucho menos hacer juicios sobre la superioridad de una sobre otra, ni discutir problemas de progreso o evolución en la historia de las teorías políticas; seríamos capaces de identificar el carácter ideológico en su contexto específico, pero no podríamos discutir el alcance cognoscitivo de una teoría, en cuanto explicación o comprensión de la realidad; podríamos, en suma, reconstruir con buen grado de aproximación el significado original de un texto del pasado, pero no podríamos hablar de su relevancia contemporánea.

Estos dilemas nos obligan a considerar estas perspectivas no como alternativas excluyentes, sino como propuestas que pueden complementarse mutuamente. Claro está que la búsqueda de esta interpretación complementaria no puede ser mecánica, sino que exige la construcción de una tercera alternativa. 


\section{Hacia una perspectiva histórico-filosófica}

Considero que puede integrarse la cuestión de validez sustantiva de las teorías políticas del pasado con el problema de validez de sus interpretaciones históricas, si se adopta un criterio histórico de validez. Este criterio ha de juzgar la validez de una determinada teoría no de acuerdo con dudosos estándares de racionalidad universal, sino desde una visión retrospectiva, en términos de su capacidad para responder a problemas teóricos y prácticos que teorías anteriores no resolvieron. Esto exige que las interpretaciones no sólo deben estar orientadas a reconstruir sincrónicamente la especificidad de una teoría determinada en su contexto particular, sino que además deben ubicar la teoría en cuestión en su desarrollo en el tiempo. Esto es, la interpretación histórica debe esforzarse por reconstruir el origen, desarrollo y evolución de la teoría política de tal manera que se pueda identificar la racionalidad implícita en su desarrollo.

Me parece que la propuesta interpretativa de Alasdair MacIntyre, que concibe el desarrollo de las teorías políticas dentro de tradiciones dinámicas de investigación, adopta un criterio evaluativo con el cual la validez de una teoría determinada exige la reconstrucción histórica de la tradición a la que pertenece, para poder apreciar la medida en que dicha teoría representa un progreso en la solución de problemas prácticos y cognoscitivos. ${ }^{18}$

De esta manera, para MacIntyre la historia de las teorías políticas no es una disciplina independiente y aparte de la evaluación crítica de las teorías sustantivas, sino que constituye el proceso de evaluación misma, ya que sin recons-

18 A. MacIntyre desarrolla su concepción de "tradiciones racionales de investigación" principalmente en su libro Whose Justice? Which Rationality?, University of Notre Dame Press, Notre Dame, 1988. 
trucción histórica no puede haber discusiones en torno al problema de la validez de la teoría. ${ }^{19}$

Convergentemente con lo anterior, la validez de la interpretación de la teoría depende de su pertinencia para develar la racionalidad presupuesta en el debate entre diferentes teorías. Desde esta perspectiva, cercana al punto de vista de Lakatos y de Laudan, MacIntyre concibe de manera interdependiente la validez de las teorías políticas sustantivas y la validez de sus interpretaciones históricas.

Este criterio histórico también nos permite superar el dilema de relevancia $v s$. significado original, ya que la interpretación objetiva y auténtica de un texto de teoría política necesariamente apunta hacia el problema de su contribución al desarrollo de la tradición a la que pertenece, o incluso de su contribución al desarrollo de otras tradiciones. Esta visión dinámica de las teorías políticas resulta superior a la perspectiva straussiana con claras tendencias a suprimir la historicidad de las teorías en aras de una universalidad dudosa y exagerada, y a la perspectiva skinneriana que se inclina por reducir la historicidad a un estático contexto social.

Desde la perspectiva diacrónica de MacIntyre, el contexto propio y auténtico de las teorías políticas no se reduce a circunstancias sociales y comunicativas, estáticas y aisladas; tampoco la relevancia y significación de las teorías presupone un conjunto de problemas y estándares universales y transhistóricos. En oposición a estas dos visiones contrarias, MacIntyre considera que el contexto histórico de una teoría es siempre una tradición en movimiento. Consecuentemente, el rescate de la historicidad de una teoría

19 "Evaluar una teoría, como evaluar una serie de teorías, un programa de investigación de Lakatos, consiste precisamente en escribir su historia, esa narrativa de sus derrotas y victorias." (A. MacIntyre, "Epistemological Crisis, Dramatic Narratives and the Philosophy of Science", Philosophy and Rhetoric, octubre, 1977, p. 469.) 
implica preguntarse por la relevancia y contribución de dicha teoría al desarrollo de la tradición. MacIntyre asigna a los conflictos internos (problemáticas) y externos (crisis epistemológicas) de las tradiciones un papel fundamental en su desarrollo y progreso:

Una tradición es una discusión que se desarrolla a través del tiempo, en la cual ciertos acuerdos fundamentales se definen en términos de dos tipos de conflictos: aquellos debates con críticos y enemigos externos a la tradición que rechazan todos los acuerdos, o al menos los principales, y aquellos conflictos internos por medio de los cuales se expresa el significado y racionalidad de los acuerdos fundamentales. El progreso en la solución de estos conflictos es el factor determinante en la consolidación de una tradición. ${ }^{20}$

Al ubicar las teorías políticas dentro de tradiciones de investigación racional que son al mismo tiempo locales y dinámicas, se puede respetar la historicidad de la teoría política sin ser anticuario, y evaluar su relevancia sin pecar de anacrónico.

El reconocimiento de debates entre tradiciones distintas supone reconocer la posibilidad de comunicación entre éstas y, por ende, implica la superación de la inconmensurabilidad entre ellas. El debate y comunicación entre tradiciones distintas no presupone criterios universales de racionalidad, ni lenguajes o conceptos transhistóricos. Para MacIntyre, estos debates requieren simplemente la posibilidad de traducir los conceptos y principios de una tradición a otra por medio de dos vías: traducción textual directa ( $s a-$ me saying translation) y traducción por innovación con-

20 A. MacIntyre, Whose Justice? Which Rationality, p. 390. Obsérvese que esta manera de concebir la dinámica de las tradiciones de investigación integra aspectos de la visión kuhniana del cambio científico (crisis epistemológicas) con la visión popperiana (continuidad y progreso mediante resolución de conflictos). 
ceptual (linguistic innovation). ${ }^{21}$ Gracias a estos dos tipos de traducción, los miembros de una tradición pueden apropiarse de contenidos teóricos de otra ajena y confrontarlos con los contenidos originales de la propia. MacIntyre reconoce la dificultad de este tipo de comunicación entre tradiciones, ya que involucra no sólo la traducción de algunos conceptos, sino en realidad el aprendizaje de todo un lenguaje extraño. Sin embargo, muy optimistamente MacIntyre considera que puede aprenderse una lengua ajena como si fuera "una segunda primera lengua".

Esta manera de concebir la comunicación entre tradiciones distintas sugiere una forma de superar el dilema relativismo o universalismo al que parece conducir una posición historicista como la de Skinner, o una posición filosófica como la de Strauss. Para MacIntyre, la confrontación de los principios y conceptos fundamentales de una tradición históricamente definida con principios y acuerdos de tradiciones ajenas (crisis epistemológicas) es condición fundamental para el reconocimiento de la racionalidad de la tradición en cuestión. ${ }^{22}$

Finalmente, en relación con la disyuntiva: ideología vs. teoría filosófica, MacIntyre también ofrece una respuesta alternativa. Este autor no identifica, como lo hace Skinner, ideología política con filosofía política, ni tampoco marca una ruptura tajante entre ambos tipos de conocimiento, como lo hace Strauss; más bien, concibe las teorías políticas como elaboraciones más o menos críticas y fundadas de ideologías políticas. En el nivel de estas elaboraciones es

${ }^{21}$ Cfr. ibid, p. 372.

22 "La existencia de amplias posibilidades de intraducibilidad [textual] y por lo tanto las posibilidades de desafío a la hegemonía cultural, lingüística, social y racional de la propia tradición [...] es más que una amenaza. Solamente aquellos en cuya tradición hay lugar para el cuestionamiento de su hegemonía pueden tener garantías racionales de su hegemonía." (Ibid., p. 388.) 
posible escudriñar, denunciar y transformar aspectos irracionales, opresivos o inmorales (de acuerdo con estándares locales) de las ideologías y prácticas políticas que en su manifestación cotidiana resultan inadvertidos. Asimismo, es posible que esta crítica racional de los elementos opresivos o irracionales de las ideologías se materialice de alguna manera en cambios en instituciones y nuevas formas de vida política que, a su vez, den origen a nuevas formas ideológicas. MacIntyre concibe las teorías políticas en constante movimiento y transformación. Para él, no todos los aspectos de una teoría son meramente ideológicos o meramente filosóficos, sino que siempre hay una mezcla de ellos; ni tampoco los elementos de un tipo lo son de manera definitiva; los aspectos filosóficos pueden materializarse y tornarse en ideologías y los aspectos ideológicos pueden ser susceptibles de elaboración crítica y racional, y transformarse en filosofías. ${ }^{23}$

Los elementos de síntesis cambiante de ideología y conocimiento teórico en sentido estricto están enmarcados en el movimiento histórico de las teorías y de sus tradiciones, y contribuyen a éste. El progreso de la teoría no sólo se determina en términos de resolución de problemas estrictamente teóricos, sino también en términos de su contribución a problemas prácticos. Desde esta perspectiva, el progreso de una teoría o de una tradición depende tanto de su contribución a la solución de enigmas filosóficos o científicos, como de problemas prácticos específicos. Lejos de ser incompatibles estos dos tipos de problemas y soluciones se complementan y refuerzan recíprocamente.

23 "Lo que considero ideología no sólo se traslapa con lo que concierne propiamente a la filosofía, sino que es filosofía. Así, la investigación filosófica es siempre capaz de convertirse en un solvente de las convicciones y compromisos ideológicos, al arribar a conclusiones incompatibles con cualquier ideología particular." (A. MacIntyre, Against the Self-Images of the Age, Duckworth, 1971, pp. 6-7.) 


\section{Hermenéutica y reconstrucción de las teorías políticas}

La perspectiva interpretativa de MacIntyre, basada en el concepto de "tradición de investigación racional", permite avanzar significativamente en la solución de los problemas a que conducen las perspectivas filosóficas o históricas consideradas en forma aislada. Sin embargo, la propuesta de MacIntyre presenta algunos serios problemas si se estudia a la luz de las teorías hermenéuticas contemporáneas.

Específicamente el problema que presenta la propuesta de MacIntyre se refiere a su tesis de que es posible entender un lenguaje ajeno como si fuera una "segunda primera lengua" y, de esta manera, comprender una tradición ajena, en sus términos originales. Esta tesis está basada en la idea de que toda expresión cultural tiene un significado original, único, que es posible recobrar sin interferencia o deformación por parte del lenguaje, cultura y prejuicios de la tradición del intérprete. En este punto, MacIntyre, al igual que Skinner y Strauss, se acercan mucho a la posición de $\mathrm{W}$. Dilthey, quien consideraba posible recuperar (revivir) el significado original de las expresiones culturales que constituyen el objeto de estudio de las ciencias del espíritu (Geisteswissenschaften). Autores hermenéuticos contemporáneos han llamado a esta pretensión "la ilusión objetivista".

Desafortunadamente, ni MacIntyre ni Skinner ${ }^{24}$ prestan suficiente atención a las objeciones a la ilusión objetivista que presentan autores de la teoría hermenéutica contemporánea (Gadamer, Ricoeur, Habermas). El punto funda-

24 Strauss mantuvo una correspondencia crítica con Gadamer, pero fue poco sensible a sus argumentos. (Cfr. correspondencia H.G. Gadamer y L. Strauss, Independent Journal of Philosophy, no. 2, 1978.) Skinner simplemente hace una referencia en términos despectivos a la concepción positiva de Gadamer de los prejuicios. (Cfr. Q. Skinner, "The Idea of Negative Liberty", en Q. Skinner et al. (comps.), Philosophy..., p. 202.) 
mental que argumentan estos autores es que el intérprete, al igual que el autor (o actor), está siempre ubicado en ciertas circunstancias sociales, culturales, comunicativas, etc., que condicionan toda interpretación. Este contexto condicionante constituye una situación hermenéutica ${ }^{25}$ del intérprete a la cual no se puede renunciar. En consecuencia, el significado que resulta de toda interpretación está necesariamente codeterminado por el autor y el intérprete, como lo afirma Gadamer:

El significado real de un texto que tiene como destinatario a un intérprete no sólo depende de los factores ocasionales que caracterizan al autor y su público original. Este significado está también codeterminado por la situación histórica del intérprete y, por lo tanto, por la totalidad del curso objetivo de la historia. ${ }^{26}$

En términos muy semejantes Paul Ricoeur subraya el carácter continuamente cambiante del significado de los textos y las acciones humanas:

Como un texto, la acción humana es una obra abierta, cuyo significado está en suspenso. Es precisamente porque la acción humana devela nuevas referencias y rescata relevancia fresca de las interpretaciones, por lo que las acciones humanas están en espera de nuevas interpretaciones que decidan su significado. ${ }^{27}$

25 Esta idea la derivan los filósofos hermenéuticos contemporáneos de Heidegger: "toda interpretación parte de pre-visiones y de preconcepciones. Si tal interpretación, en cuanto Interpretación [auténtica] es una tarea de investigación, entonces la explicitación de todos esos presupuestos (que llamamos 'situación hermenéutica'), necesita esclarecerse y asegurarse de antemano." (M. Heidegger, Being and Time, Harper and Row, Nueva York, 1967, sec. 45, p. 275.)

26 H.G. Gadamer, Philosophical Hermeneutics, editado por E. Ling, University of California Press, Los Ángeles, 1977, p. XXV.

27 Paul Ricoeur, "The Model of the Text: Meaningful Action as 
Dado el hecho de que el significado de los textos y las acciones está en continua reconstrucción, debido a las diversas interpretaciones de que son objeto, la tarea de buscar y rescatar un significado original en los términos propios del autor es una tarea quimérica, una "ilusión objetivista". Si se toma en serio la tesis contextualista de que el autor de un texto escribió su obra en condiciones comunicativas, culturales, ideológicas y sociales determinadas, no hay razón por la cual privilegiar al intérprete como un sujeto libre e independiente de sus circunstancias históricas, de su situación hermenéutica. Por el contrario, la tesis contextualista debe ser simétrica, esto es, debe regir tanto para el autor como para el intérprete.

Los tres autores que hemos discutido no toman de manera simétrica la tesis del condicionamiento histórico, sino que injustificadamente la aplican al autor y no al intérprete. Quizá la razón de esta inconsistencia sea un exagerado optimismo metodológico, herencia de las concepciones empiristas de las ciencias sociales que postulan como principio metodológico la neutralidad valorativa del científico. En todo caso, es difícil de explicar el poco interés de estos historiadores de las teorías políticas por entablar un diálogo serio y abierto con autores como Gadamer y Ricoeur, que argumentan en contra de la posibilidad de neutralidad del intérprete.

Considero que una perspectiva de interpretación de las teorías políticas que realmente integre los cuestionamientos filosóficos e históricos y que supere los dilemas que hemos planteado requiere de una seria fundamentación hermenéutica. En particular, la tesis simétrica del condicionamiento histórico es un principio hermenéutico que al menos debe

a Text", en Fred Dalhmayr y Thomas McCarthy (comps.), Understanding and Social Inquiry, University of Notre Dame Press, 1977, p. 327. 
ser discutido seriamente por filósofos e historiadores de las teorías políticas. Esta tesis implica abandonar la "ilusión objetivista" del significado original y, consecuentemente, conduce a renunciar a los criterios correspondentistas de validez presupuestos en las metodologías como las de Skinner y MacIntyre.

En lugar de este tipo de criterio empirista de validez la tesis hermenéutica de la simetría apunta hacia una noción de validez fundada en el poder heurístico de las interpretaciones para develar potencialidades de las teorías que se interpretan para su ulterior desarrollo en contextos diferentes a los del autor.

Esta noción de validez ciertamente resulta más congruente con la perspectiva histórica que defiende MacIntyre. Recordemos que en esta perspectiva, la validez de la interpretación de una teoría depende de su capacidad heurística para develar la racionalidad presupuesta en el debate con otras teorías en competencia. Esta racionalidad sólo puede apreciarse retrospectivamente al evaluar el cambio de las teorías dentro de la tradición y la contribución de esos cambios al progreso mismo de la tradición. Este criterio histórico de validez presupone una noción de significado siempre cambiante y móvil, siempre en progreso, que resulta incompatible con la noción de significado original y con el criterio de validez como correspondencia o adecuación del significado interpretado respecto a un supuesto significado original del texto.

\section{Conclusiones}

A partir de la discusión anterior, quisiera simplemente subrayar algunos puntos básicos para integrar la reflexión filosófica y el análisis histórico en la interpretación de las teorías políticas del pasado de tal manera que puedan superarse los problemas y dilemas que hemos señalado. 
a) La interpretación de las teorías políticas del pasado requiere tanto del análisis sincrónico como de reconstrucciones retrospectivas. Esto implica que la interpretación de los textos debe procurar reconstruir el significado de la teoría misma, así como la significación o relevancia de ésta en contextos ideológicos y teóricos diferentes. De hecho, es importante reconocer que la cambiante significación de los textos conduce a un cambio continuo de su significado. Así por ejemplo, las interpretaciones republicanistas de la obra de Maquiavelo, desde la tradición democrática anglosajona, conducen a asignar a dicha obra un significado completamente opuesto a las interpretaciones que la ubican como fundadora del moderno realismo político.

b) De acuerdo con el primer punto, es indispensable reconocer el carácter cambiante y no fijo del significado de los textos y, por lo tanto, rechazar la "ilusión objetivista" de que los textos tienen un significado original y único que está en espera de ser recuperado por el intérprete mediante una metodología apropiada. Por el contrario, es importante asumir que toda interpretación es una recreación del significado del texto, y por ello toda interpretación cambia su significado (Gadamer, Ricoeur).

c) El punto anterior no es meramente resultado de una decisión metodológica, sino efecto y consecuencia del hecho de que tanto el intérprete como el autor están sujetos a circunstancias y contextos específicos (tesis simétrica del condicionamiento). Este hecho constituye una explicación y una justificación de los dos puntos anteriores: si los contextos históricos del autor y del intérprete son constitutivos del autor en cuanto autor y del intérprete en cuanto tal, los efectos condicionantes de estos contextos no pueden ser eliminados o suspendidos ni en la escritura de la obra ni en la interpretación de la misma. Los contextos y las intenciones de autor e intérprete codeterminan el significado de 
la obra, y si los contextos de las interpretaciones cambian en el tiempo, el significado de la obra también cambia. El análisis sincrónico privilegiará la parte del significado determinado por el autor, mientras que el diacrónico pondrá énfasis en el punto de vista del intérprete, de su contexto y de la historia habida entre el autor y el intérprete.

d) La tesis del condicionamiento simétrico del intérprete y del autor no involucra de manera alguna una posición sociológica determinista, en el sentido de que las teorías políticas son mero reflejo y expresión de estructuras sociales y relaciones de poder. Sin negar que las teorías políticas son en buena medida ideologías, también se debe reconocer, como lo hace MacIntyre, que tienen contenidos teóricos en sentido estricto, esto es, constituyen conocimiento acerca de hechos y procesos políticos y contienen evaluaciones acerca de esos hechos fundados en argumentos racionales. Es más, en buena medida las teorías políticas no sólo contienen conocimiento acerca de los hechos y situaciones de su tiempo, sino que también pretenden sustentar conocimiento de regularidades o de estructuras transhistóricas. Si se acepta este carácter de las teorías políticas, tanto el análisis textual, como el contextual son indispensables en el estudio sincrónico y diacrónico de las teorías políticas. Sin establecer una separación tajante, el análisis textual pondría énfasis en los contenidos propiamente cognoscitivos de las teorías, mientras que el examen contextual de los textos apuntaría más a los aspectos ideológicos. Desde luego, los contenidos ideológicos pueden tornarse en teóricos mediante la racionalización y examen crítico de las ideologías o, a la inversa, al institucionalizar consecuencias de los contenidos propiamente teóricos, se pueden generar concepciones ideológicas.

e) El punto b), relativo al carácter cambiante del significado, y el punto d) referente al carácter ideológico, además 
del propiamente teórico de las teorías políticas, parecerían conducir a un relativismo historicista. Sin embargo, esto no es así si se adopta un criterio heurístico para juzgar la validez de las interpretaciones, en términos de los "nuevos" aspectos que la interpretación devela. Con este criterio, es posible mantener la tesis del significado cambiante y, al mismo tiempo, poder decidir sobre interpretaciones en competencia. Esta evaluación habría de tomarse tanto en términos de la información que se ofrece con relación al contexto teórico, ideológico y cultural del autor, como de la reconstrucción que se haga de la obra en una visión retrospectiva desde el contexto del intérprete. En estos dos niveles es importante considerar la manera en que la obra en cuestión resuelve problemas teóricos y prácticos en el tiempo del autor y también las contribuciones de la obra al desarrollo histórico de la disciplina y las repercusiones en contextos ideológicos y culturales diferentes a las del autor.

Desde esta perspectiva, las interpretaciones enriquecen los contenidos de las teorías mismas, al develar contribuciones y repercusiones teóricas e ideológicas que el autor mismo de la obra no contempló, pero que forman parte de la teoría misma. Por ello, al evaluar de esta manera las interpretaciones de las teorías políticas, también se evalúan los contenidos sustantivos de tales teorías. 


\section{SUMMARY}

This work belongs to the discussion between historians and science philosophers on methodological matters in the study of the nature and development of scientific theories. Notably, this paper seeks to state some fundamental steps towards the interpretation and reconstruction of political theories. In order to achieve this, Leo Strauss's philosophical view, as well as Quentin Skinner's historical approach, are critically examined, pointing out their most significant progresses as well as their main problems and weaknesses. On the grounds of this critical analysis, the need to seriously consider the history and philosophy of political theory as being mutually complementary is stated. Within this comprehensive approach, this paper considers the proposal of Alasdair MacIntyre which seeks to recover, through the concept of "research tradition", significant philosophical and historical studies within political theory. Notwithstanding, MacIntyre, Strauss and Skinner do not take contemporary hermeneutic theories seriously when they formulate their interpretative perspectives. Beginning with contemporary hermeneutic philosophers (Gadamer, Ricoeur), the most significant contributions by Strauss, Skinner and MacIntyre are looked at in order to develop a historical and philosophical approach to study change and evolution in political theories.

[Traducción: Gabriela Montes de Oca Vega] 